\title{
Some Common Fixed Point Theorems in Generalized Metric Spaces
}

\author{
Manoj Kumar, ${ }^{1,2}$ Pankaj Kumar, ${ }^{3}$ and Sanjay Kumar ${ }^{4}$ \\ ${ }^{1}$ Department of Mathematics, Delhi Institute of Technology and Management, Gannaur 131101, Sonipat, India \\ ${ }^{2}$ Guru Jambheshwar University of Science and Technology, Hisar 125001, India \\ ${ }^{3}$ Department of Mathematics, Guru Jambheshwar University of Science and Technology, Hisar 125001, India \\ ${ }^{4}$ Department of Mathematics, Deenbandhu Chhotu Ram University of Science and Technology, Murthal 131039, India
}

Correspondence should be addressed to Sanjay Kumar; sanjaymudgal2004@yahoo.com

Received 30 January 2013; Accepted 18 March 2013

Academic Editor: Jen-Chih Yao

Copyright (C) 2013 Manoj Kumar et al. This is an open access article distributed under the Creative Commons Attribution License, which permits unrestricted use, distribution, and reproduction in any medium, provided the original work is properly cited.

First we prove common fixed point theorems for weakly compatible maps which generalize the results of Chen (2012). Secondly, we prove common fixed point theorems using property E.A. along with weakly compatible maps. At the end, we prove common fixed point theorems using common limit range property (CLR property) along with weakly compatible maps.

\section{Introduction}

In 2000, Branciari [1] introduced the notion of generalized metric space as follows.

Definition 1. Let $X$ be a nonempty set and let $d: X \times X \rightarrow$ $[0, \infty)$ be a mapping such that for all $x, y \in X$ and for all distinct points $u, v \in X$ each of them different from $x$ and $y$, one has the following:

(i) $d(x, y)=0$ if and only if $x=y$,

(ii) $d(x, y)=d(y, x)$,

(iii) $d(x, y) \leq d(x, u)+d(u, v)+d(v, y)$ (rectangular inequality).

Then $(X, d)$ is called a generalized metric space (or shortly g.m.s.).

Definition 2. Let $(X, d)$ be a g.m.s. Let $\left\{x_{n}\right\}$ be a sequence in $X$ and $x \in X$. We say that $\left\{x_{n}\right\}$ is

(i) g.m.s. convergent to $x$ if and only if $d\left(x_{n}, x\right) \rightarrow 0$ as $n \rightarrow \infty$,

(ii) g.m.s. Cauchy sequence if and only if, for each $\varepsilon>0$, there exists $n_{0} \in \mathbb{N}$ such that, $d\left(x_{m}, x_{n}\right)<\varepsilon$ for all $n>m>n_{0}$ (iii) complete g.m.s. if every g.m.s. Cauchy sequence is g.m.s. convergent in $X$.

In 1986, Jungck [2] introduced the notion of compatible maps as follows.

Let $(X, d)$ be a metric space. Let $S, F: X \rightarrow X$ be two single-valued functions. We say that $S$ and $F$ are compatible if $\lim _{n \rightarrow \infty} d\left(S F x_{n}, F S x_{n}\right)=0$, whenever $\left\{x_{n}\right\}$ is a sequence in $X$ such that $\lim _{n \rightarrow \infty} d\left(F x_{n}, S x_{n}\right)=0$.

In 1998, Jungck and Rhoades [3] introduced the notion of weakly compatible maps as follows.

Two maps are said to be weakly compatible if they commute at the coincidence point. Note that compatible maps are weakly compatible but the converse is not true in general.

In 2002, Aamri and El Moutawakil [4] introduced the notion of property E.A. as follows.

Let $A$ and $B$ be two self-mappings of a metric space $(X, d)$. We say that $A$ and $B$ satisfy the property E.A. if there exists a sequence $\left\{x_{n}\right\}$ such that $\lim _{n \rightarrow \infty} A x_{n}=\lim _{n \rightarrow \infty} B x_{n}=t$ for some $t$ in $X$.

In 2011, Sintunavarat and Kumam [5] introduced the notion of (CLRg) property as follows. 
Let $(X, d)$ be a metric space. Two mappings $f: X \rightarrow X$ and $g: X \rightarrow X$ are said to satisfy (CLRg) property if there exists sequences $\left\{x_{n}\right\}$ in $X$ such that

$$
\lim _{n \rightarrow \infty} f\left(x_{n}\right)=\lim _{n \rightarrow \infty} g\left(x_{n}\right)=g(t) \quad \text { for some } t \text { in } X \text {. }
$$

Similarly, we can state $\left(\mathrm{CLR}_{F}\right)$ and $\left(\mathrm{CLR}_{G}\right)$ properties if the mapping $g: X \rightarrow X$ has been replaced by the mapping $F$ : $X \rightarrow X$ and $G: X \rightarrow X$, respectively.

In 2012, Chen [6] introduced the notion of $\mathscr{W}$ function and $\mathcal{S}$ function as follows.

Definition 3. A function $\varphi: \mathbb{R}^{+} \rightarrow \mathbb{R}^{+}$is said to be a $\mathscr{W}$ function if the function $\phi$ satisfies the following conditions:

$$
\begin{aligned}
& \left(\phi_{1}\right) \phi(t)<t \text { for all } t>0 \text { and } \phi(0)=0, \\
& \left(\phi_{2}\right) \lim _{t_{n \rightarrow t}} \inf \phi\left(t_{n}\right)<t \text { for all } t>0 .
\end{aligned}
$$

Definition 4. A function $\varphi: \mathbb{R}^{+} \rightarrow \mathbb{R}^{+}$is said to be a $\delta$ function if the function $\phi$ satisfies the following conditions:

$\left(\phi_{1}\right) \phi$ is a strictly increasing, continuous function in each coordinate,

$\left(\phi_{2}\right)$ for all $t>0, \phi(t, t, t)<t, \phi(t, 0,0)<t, \phi(0, t, 0)<$ $t$, and $\phi(0,0, t)<t$.

Before proving our results, we need the following lemma.

Lemma 5 (see [6]). Let $\phi: \mathbb{R}^{+} \rightarrow \mathbb{R}^{+}$be a $\mathscr{W}$ function. Then $\lim _{n \rightarrow \infty} \phi^{n}(t)=0$ for all $t>0$, where $\phi^{n}(t)$ denotes the $n$th iteration of $\phi$.

\section{Weakly Compatible Maps}

In 2012, Chen [6] proved the following fixed point theorems for compatible maps.

Theorem 6. Let $(X, d)$ be Hausdorff and complete g.m.s. and let $\phi: \mathbb{R}^{+} \rightarrow \mathbb{R}^{+}$be a $\mathscr{W}$ function. Let $S, T, F$, and $G$ be selfmaps on $X$ satisfying the following:

$$
\begin{array}{rr}
T(X) \subseteq F(X), \quad S(X) \subseteq G(X), & \\
d(S x, T y) \leq \phi(\max \{d(F x, G y), d(F x, S x), d(G y, T y)\}) \\
\forall x, y \in X,
\end{array}
$$

pairs $\{S, F\}$ and $\{T, G\}$ are compatible.

If $F$ or $G$ is continuous, then $S, T, F$, and $G$ have a unique common fixed point in $X$.

Theorem 7. Let $(X, d)$ be Hausdorff and complete g.m.s. and let $\phi: \mathbb{R}^{+3} \rightarrow \mathbb{R}^{+}$be a $\mathcal{S}$ function. Let $S, T, F$, and $G$ be self-maps on $X$ satisfying (2), (4), and the following:

$$
\begin{aligned}
& d(S x, T y) \\
& \quad \leq \phi(\{d(F x, G y), d(F x, S x), d(G y, T y)\}) \quad \forall x, y \in X .
\end{aligned}
$$

Therefore, $S v=u=F v$ implies that the pair $(S, F)$ has a point of coincidence.

As $S(X) \subseteq G(X), S v=u$ implies that $u \in G(X)$. Letting $w \in G^{-1}(u)$, then $G w=u$.

Now, from (3), we have

$$
\begin{gathered}
d\left(z_{2 n}, T w\right)=d\left(S x_{2 n}, T w\right) \\
\leq \phi\left(\operatorname { m a x } \left\{d\left(F x_{2 n}, G w\right), d\left(F x_{2 n}, S x_{2 n}\right),\right.\right. \\
d(G w, T w)\}) .
\end{gathered}
$$

Letting $n \rightarrow \infty$, we have

$$
\begin{aligned}
d(u, T w) & \leq \phi(\max \{d(u, u), d(u, u), d(u, T w)\}) \\
& =\phi(d(u, T w))<d(u, T w), \text { a contradiction. }
\end{aligned}
$$

(5) Therefore, $u=T w$. 
Thus, we have shown that $u=S v=F v=T w=G w$, which amounts to say that both pairs have point of coincidence. If one assumes $G(X)$ to be complete, then an analogous argument establishes this claim.

The remaining two cases pertain essentially to the previous cases. Indeed, if $S(X)$ is complete, then $u \in S(X) \subseteq G(X)$, and if $T(X)$ is complete, then $u \in T(X) \subseteq F(X)$. Thus $S$ and $F, T$, and $G$ have a point of coincidence.

Since the pairs $(S, F)$ and $(T, G)$ are weakly compatible at $v$ and $w$, respectively, then

$$
\begin{gathered}
S u=S(F v)=F(S v)=F u, \\
T u=T(G w)=G(T w)=G u .
\end{gathered}
$$

If $S u \neq u$, then

$$
\begin{aligned}
d(S u, u) & =d(S u, T w) \\
& \leq \phi(\max \{d(F u, G w), d(F u, S u), d(G w, T w)\}) \\
& =\phi(\max \{d(S u, u), 0,0\}) \\
& =\phi(d(S u, u))<d(S u, u), \text { a contradiction. }
\end{aligned}
$$

Therefore, $S u=u$.

Similarly, one can show that $T u=u$.

Thus $u$ is the common fixed point of $S, T, F$, and $G$.

Finally, we prove that $S, T, F$, and $G$ have a unique common fixed point. Let $t$ be another common fixed point of $S, T, F$, and $G$.

From (3), we have

$$
\begin{aligned}
d(t, u) & =d(S t, T u) \\
& \leq \phi(\max \{d(F t, G u), d(F t, S t), d(G u, T u)\}) \\
& =\phi(\max \{d(t, u), d(t, t), d(u, u)\}) \\
& <d(t, u), \quad \text { a contradiction unless } d(t, u)=0, \\
& \text { that is, } t=u .
\end{aligned}
$$

Hence $u$ is the unique common fixed point of $S, T, F$, and $G$ in $X$.

We give the following example to illustrate Theorem 8 .

Example 9. Let $X=\left\{a_{1}, a_{2}, a_{3}, a_{4}, a_{5}\right\}$, where $a_{1}, a_{2}, a_{3}, a_{4}$, and $a_{5}$ are positive constants. We define $d: X \times X \rightarrow[0, \infty)$ by

(1) $d(x, x)=0$, for all $x \in X$,

(2) $d(x, y)=d(y, x)$, for all $x, y \in X$,

(3) $d\left(a_{1}, a_{2}\right)=3 k$,

(4) $d\left(a_{1}, a_{3}\right)=d\left(a_{2}, a_{3}\right)=k$,

(5) $d\left(a_{1}, a_{4}\right)=d\left(a_{2}, a_{4}\right)=d\left(a_{3}, a_{4}\right)=2 k$,

(6) $d\left(a_{1}, a_{5}\right)=d\left(a_{2}, a_{5}\right)=d\left(a_{3}, a_{5}\right)=d\left(a_{4}, a_{5}\right)=2 k$,

where $k>0$ is a constant.
If $\phi: \mathbb{R}^{+} \rightarrow \mathbb{R}^{+}, \phi(t)=(2 / 3) t$, then $\phi$ is a $\mathscr{W}$ function. We next define $S, T, F, G: X \rightarrow X$ by

$$
\begin{aligned}
& S(x)= \begin{cases}a_{3}, & \text { if } x \neq a_{4}, \\
a_{5}, & \text { if } x=a_{4},\end{cases} \\
& T(x)= \begin{cases}a_{3}, & \text { if } x \neq a_{4}, \\
a_{1}, & \text { if } x=a_{4},\end{cases}
\end{aligned}
$$

$G(x)=I(x)=$ the identity mapping,

$$
F(x)= \begin{cases}a_{3}, & \text { if } x=a_{3}, \\ a_{1}, & \text { if } x=a_{1}, a_{2}, a_{5}, \\ a_{2}, & \text { if } x=a_{4} .\end{cases}
$$

Then all the conditions of Theorem 8 are satisfied and $a_{3}$ is a unique common fixed point of $S, T, F$, and $G$.

Theorem 10. Let $(X, d)$ be Hausdorff and complete g.m.s. and let $\phi: \mathbb{R}^{+3} \rightarrow \mathbb{R}^{+}$be a $\mathcal{S}$ function. Let $S, T, F$, and $G$ be selfmaps on $X$ satisfying (2), (5) and (6). If one of $S(X), T(X)$, $F(X)$, and $G(X)$ is a complete subset of $X$, then $S, T, F$, and $G$ have a unique common fixed point in $X$.

Proof. Given that $x_{0} \in X$, define the sequence $\left\{x_{n}\right\}$ recursively as follows:

$$
\begin{gathered}
G x_{2 n+1}=S x_{2 n}=z_{2 n}, \\
F x_{2 n+2}=T x_{2 n+1}=z_{2 n+1} .
\end{gathered}
$$

Then due to Theorem 7 [6], $\left\{z_{n}\right\}$ is g.m.s. Cauchy sequence in $X$. Now, suppose that $F(X)$ is complete subset of $X$; then the subsequence $z_{2 n+1}=F x_{2 n+2}$ must get a limit in $F(X)$. Call it to be $u$ and $v \in F^{-1}(u)$. Then $F(v)=u$. As $\left\{z_{n}\right\}$ is a Cauchy sequence containing a convergent subsequence $\left\{z_{2 n+1}\right\}$, therefore the sequence $\left\{z_{n}\right\}$ also converges implying thereby the convergence of $\left\{z_{2 n}\right\}$ being a subsequence of the convergent sequence $\left\{z_{n}\right\}$. On taking $x=v$ and $y=x_{2 n+1}$ in (5) one gets

$$
\begin{aligned}
& d\left(S v, T x_{2 n+1}\right) \\
& \quad \leq \phi\left(\left\{d\left(F v, G x_{2 n+1}\right), d(F v, S v), d\left(G x_{2 n+1}, T x_{2 n+1}\right)\right\}\right) .
\end{aligned}
$$

Letting $n \rightarrow \infty$, we have

$$
\begin{aligned}
d(S v, u) & \leq \phi(\{d(u, u), d(u, S v), d(u, u)\}) \\
& =\phi(d(u, S v))<d(S v, u), \text { a contradiction. }
\end{aligned}
$$

Therefore, $S v=u=F v$, which shows that the pair $(S, F)$ has a point of coincidence.

As $S(X) \subseteq G(X), S v=u$ implies that $u \in G(X)$. Letting $w \in G^{-1}(u)$, then $G w=u$.

From (5), we have

$$
\begin{aligned}
d\left(z_{2 n}, T w\right)= & d\left(S x_{2 n}, T w\right) \\
\leq & \phi\left(\left\{d\left(F x_{2 n}, G w\right), d\left(F x_{2 n}, S x_{2 n}\right),\right.\right. \\
& d(G w, T w)\}) .
\end{aligned}
$$


Letting $n \rightarrow \infty$, we have

$$
\begin{aligned}
d(u, T w) & \leq \phi(\{d(u, u), d(u, u), d(u, T w)\}) \\
& =\phi(d(u, T w))<d(u, T w), \text { a contradiction. }
\end{aligned}
$$

Therefore, $u=T w$. Thus, we have shown that $u=S v=F v=$ $T w=G w$, which amounts to say that both pairs have point of coincidence. If one assumes $G(X)$ to be complete, then an analogous argument establishes this claim.

The remaining two cases pertain essentially to the previous cases. Indeed if $S(X)$ is complete, then $u \in S(X) \subseteq G(X)$, and if $T(X)$ is complete, then $u \in T(X) \subseteq F(X)$.

Thus $S$ and $F, T$, and $G$ have a point of coincidence.

Since the pairs $(S, F)$ and $(T, G)$ are weakly compatible at $v$ and $w$, respectively, then

$$
\begin{gathered}
S u=S(F v)=F(S v)=F u, \\
T u=T(G w)=G(T w)=G u .
\end{gathered}
$$

If $S u \neq u$, then

$$
\begin{aligned}
d(S u, u) & =d(S u, T w) \\
& \leq \phi(\{d(F u, G w), d(F u, S u), d(G w, T w)\}) \\
& =\phi(\{d(S u, u), 0,0\}) \\
& =\phi(d(S u, u))<d(S u, u), \text { a contradiction. }
\end{aligned}
$$

Therefore, $S u=u$.

Similarly, one can show that $T u=u$.

Thus $u$ is the common fixed point of $S, T, F$, and $G$.

Finally, we prove that $S, T, F$, and $G$ have a unique common fixed point. Let $t$ be another common fixed point of $S$, $T, F$, and $G$. Then using (5), we have

$$
\begin{aligned}
& d(t, u)=d(S t, T u) \\
& \leq \phi(\{d(F t, G u), d(F t, S t), d(G u, T u)\}) \\
&=\phi(\{d(t, u), d(t, t), d(u, u)\}) \\
&<d(t, u), \text { a contradiction unless } d(t, u)=0, \\
& \text { that is, } t=u .
\end{aligned}
$$

Hence $u$ is the unique common fixed point of $S, T, F$, and $G$ in $X$.

We give the following example to illustrate Theorem 10.

Example 11. Let $X=\left\{a_{1}, a_{2}, a_{3}, a_{4}, a_{5}\right\}$, where $a_{1}, a_{2}, a_{3}, a_{4}$, and $a_{5}$ are positive constants. We define $d: X \times X \rightarrow[0, \infty)$ by

(1) $d(x, x)=0$, for all $x \in X$,

(2) $d(x, y)=d(y, x)$, for all $x, y \in X$,

(3) $d\left(a_{1}, a_{2}\right)=3 k$,

(4) $d\left(a_{1}, a_{3}\right)=d\left(a_{2}, a_{3}\right)=k$,
(5) $d\left(a_{1}, a_{4}\right)=d\left(a_{2}, a_{4}\right)=d\left(a_{3}, a_{4}\right)=2 k$,

(6) $d\left(a_{1}, a_{5}\right)=d\left(a_{2}, a_{5}\right)=d\left(a_{3}, a_{5}\right)=d\left(a_{4}, a_{5}\right)=2 k$,

where $k>0$ is a constant.

If $\phi: \mathbb{R}^{+3} \rightarrow \mathbb{R}^{+}, \phi(t)=(3 / 5) \cdot \max \left\{t_{1}, t_{2}, t_{3}\right\}$, then $\phi$ is a $\mathcal{S}$ function. We next define $S, T, F, G: X \rightarrow X$ by

$$
\begin{aligned}
& S(x)= \begin{cases}a_{3}, & \text { if } x \neq a_{4}, \\
a_{5}, & \text { if } x=a_{4},\end{cases} \\
& T(x)= \begin{cases}a_{3}, & \text { if } x \neq a_{4}, \\
a_{1}, & \text { if } x=a_{4},\end{cases}
\end{aligned}
$$

$G(x)=I(x)=$ the identity mapping,

$$
F(x)= \begin{cases}a_{3}, & \text { if } x=a_{3}, \\ a_{1}, & \text { if } x=a_{1}, a_{2}, a_{5}, \\ a_{2}, & \text { if } x=a_{4} .\end{cases}
$$

Then all the conditions of Theorem 10 are satisfied and $a_{3}$ is a unique common fixed point of $S, T, F$, and $G$.

\section{Property E.A.}

Theorem 12. Let $(X, d)$ be Hausdorff and complete g.m.s. and let $\phi: \mathbb{R}^{+} \rightarrow \mathbb{R}^{+}$be a $\mathscr{W}$ function. Let $S, T, F$, and $G$ be selfmaps on $X$ satisfying (2), (3), (6), and the following:

$\{S, F\}$ or $\{T, G\}$ satisfy the property E.A.

If the range of one of the maps $S, T, F$, or $G$ is a complete subspace of $X$, then $S, T, F$, and $G$ have a unique common fixed point in $X$.

Proof. If the pair $\{T, G\}$ satisfies the property E.A., then there exists a sequence $\left\{x_{n}\right\}$ in $X$ such that $T x_{n} \rightarrow z$ and $G x_{n} \rightarrow z$ for some $z \in X$ as $n \rightarrow \infty$. Since $T(X) \subseteq F(X)$, there exists a sequence $\left\{y_{n}\right\}$ in $X$ such that $T x_{n}=F y_{n}$. Hence $F y_{n} \rightarrow z$ as $n \rightarrow \infty$. Also, since $S(X) \subseteq G(X)$, there exists a sequence $\left\{y_{n}^{\prime}\right\}$ in $X$ such that $S y_{n}^{\prime}=G y_{n}^{\prime}$. Hence $S y_{n}^{\prime} \rightarrow z$ as $n \rightarrow \infty$.

Suppose that $F(X)$ is a complete subspace of $X$. Then, $z=$ $F u$ for some $u \in X$.

Subsequently, we have $S y_{n}^{\prime} \rightarrow F u, T x_{n} \rightarrow F u, F y_{n} \rightarrow$ $\mathrm{Fu}$ as $n \rightarrow \infty$.

From (3), we have

$$
\begin{aligned}
& d\left(S u, T x_{n}\right) \\
& \quad \leq \phi\left(\max \left\{d\left(F u, G x_{n}\right), d(F u, S u), d\left(G x_{n}, T x_{n}\right)\right\}\right) .
\end{aligned}
$$

Letting $n \rightarrow \infty$, we have

$$
\begin{aligned}
d(S u, F u) & \leq \phi(\max \{d(F u, F u), d(F u, S u), d(F u, F u)\}) \\
& =\phi(d(F u, S u))<d(F u, S u)
\end{aligned}
$$

which implies that $F u=S u$. 
The weak compatibility of $F$ and $S$ implies that $F S u=S F u$ and then $S S u=S F u=F S u=F F u$.

On the other hand, since $S(X) \subseteq G(X)$, there exists a $v \in$ $X$ such that $S u=G v$.

Now, we show that $G v=T v$.

From (3), we have

$$
\begin{gathered}
d(S u, T v) \leq \phi(\max \{d(F u, G v), d(F u, S u), d(G v, T v)\}) \\
=\phi(d(G v, T v))<d(G v, T v), \\
\text { implies that } G v=T v,
\end{gathered}
$$$$
\text { that is, } S u=F u=G v=T v \text {. }
$$

The weak compatibility of $T$ and $G$ implies that $T G v=G T v$ and $G G v=G T v=T G v=T T v$.

Let us show that $S u$ is the common fixed point of $S$, $T, F$, and $G$.

From (3), we have

$d(S S u, T v)$

$$
\begin{aligned}
\leq & \phi(\max \{d(F S u, G v), d(F S u, S S u), d(G v, T v)\}) \\
= & \phi(d(S S u, T v))<d(G v, T v) \\
& \text { implies that } S S u=T v=S u
\end{aligned}
$$

Therefore, $S u=S S u=F S u$ and $S u$ is the common fixed point of $S$ and $F$.

Similarly, we can prove that $T v$ is the common fixed point of $T$ and $G$.

Since $S u=T v$, we conclude that $S u$ is the common fixed point of $S, T, F$, and $G$.

The proof is similar when $G(X)$ is assumed to be complete subspace of $X$. The cases in which $S(X)$ or $T(X)$ is a complete subspace of $X$ are similar to the cases in which $G(X)$ or $F(X)$, respectively, is complete since

$$
S(X) \subseteq G(X), \quad T(X) \subseteq F(X) .
$$

If $S u=T u=F u=G u=u$ and $S v=T v=F v=G v=v$, then (3) gives

$$
\begin{aligned}
d(S u, T v) & \leq \phi(\max \{d(F u, G v), d(F u, S u), d(G v, T v)\}) \\
& <d(F u, G v), \quad \text { implies that } u=v
\end{aligned}
$$

Therefore, $u=v$ and the common fixed point is unique.

Theorem 13. Let $(X, d)$ be Hausdorff and complete g.m.s. and let $\phi: \mathbb{R}^{+3} \rightarrow \mathbb{R}^{+}$be a $\mathcal{S}$ function. Let $S, T, F$, and $G$ be selfmaps on $X$ satisfying (2), (5), (6), and (26). If the range of one of the maps $S, T, F$, or $G$ is a complete subspace of $X$, then $S$, $T, F$, and $G$ have a unique common fixed point in $X$.

Proof. If the pair $\{T, G\}$ satisfies the property E.A., then there exists a sequence $\left\{x_{n}\right\}$ in $X$ such that $T x_{n} \rightarrow z$ and $G x_{n} \rightarrow z$ for some $z \in X$ as $n \rightarrow \infty$.
Since $T(X) \subseteq F(X)$, there exists a sequence $\left\{y_{n}\right\}$ in $X$ such that $T x_{n}=F y_{n}$. Hence $F y_{n} \rightarrow z$ as $n \rightarrow \infty$. Also, since $S(X) \subseteq G(X)$, there exists a sequence $\left\{y_{n}^{\prime}\right\}$ in $X$ such that $S y_{n}^{\prime}=G y_{n}^{\prime}$. Hence $S y_{n}^{\prime} \rightarrow z$ as $n \rightarrow \infty$.

Suppose that $F(X)$ is a complete subspace of $X$. Then, $z=$ $F u$ for some $u \in X$.

Subsequently, we have $S y_{n}^{\prime} \rightarrow F u, T x_{n} \rightarrow F u, F y_{n} \rightarrow$ $\mathrm{Fu}$ as $n \rightarrow \infty$.

From (5), we have

$$
d\left(S u, T x_{n}\right) \leq \phi\left(d\left(F u, G x_{n}\right), d(F u, S u), d\left(G x_{n}, T x_{n}\right)\right)
$$

Letting $n \rightarrow \infty$, we have

$$
\begin{gathered}
d(S u, F u) \leq \phi(d(F u, F u), d(F u, S u), d(F u, F u)) \\
=\phi(0, d(F u, S u), 0)<d(F u, S u) \\
\text { implies that, } F u=S u .
\end{gathered}
$$

The weak compatibility of $F$ and $S$ implies that $F S u=S F u$ and then $S S u=S F u=F S u=F F u$.

On the other hand, since $S(X) \subseteq G(X)$, there exists a $v \in$ $X$ such that $S u=G v$.

Now, we show that $G v=T v$.

From (5), we have

$$
\begin{gathered}
d(S u, T v) \leq \phi(d(F u, G v), d(F u, S u), d(G v, T v)) \\
=\phi(0,0, d(G v, T v))<d(G v, T v), \\
\text { implies that } G v=T v, \\
\text { that is, } S u=F u=G v=T v .
\end{gathered}
$$

The weak compatibility of $T$ and $G$ implies that $T G v=G T v$ and $G G v=G T v=T G v=T T v$.

Let us show that $S u$ is the common fixed point of $S, T, F$ and $G$.

From (5), we have

$$
\begin{gathered}
d(S S u, T v) \leq \phi(d(F S u, G v), d(F S u, S S u), d(G v, T v)) \\
=\phi(d(S S u, T v), 0,0)<d(S S u, T v) \\
\text { implies that } S S u=T v=S u, \\
\text { that is, Su }=S S u=F S u .
\end{gathered}
$$

Therefore, $S u$ is the common fixed point of $S$ and $F$.

Similarly, one can prove that $T v$ is the common fixed point of $T$ and $G$.

Since $S u=T v$, we conclude that $S u$ is the common fixed point of $S, T, F$, and $G$.

The proof is similar when $G(X)$ is assumed to be complete subspace of $X$. The cases in which $S(X)$ or $T(X)$ is a complete subspace of $X$ are similar to the cases in which $G(X)$ or $F(X)$, respectively, is complete since $S(X) \subseteq G(X)$ and $T(X) \subseteq$ $F(X)$. 
If $S u=T u=F u=G u=u$ and $S v=T v=F v=G v=v$, then (5) gives

$$
\begin{aligned}
d(S u, T v) & \leq \phi(d(F u, G v), d(F u, S u), d(G v, T v)) \\
& <d(F u, G v), \text { implies that } u=v .
\end{aligned}
$$

Therefore, $u=v$ and the common fixed point is unique.

\section{4. (CLR) Property}

Theorem 14. Let $(X, d)$ be Hausdorff and complete g.m.s. and let $\phi: \mathbb{R}^{+} \rightarrow \mathbb{R}^{+}$be a $\mathscr{W}$ function. Let $S, T, F$, and $G$ be self-maps on $X$ satisfying (3), (6), and the following:

$T(X) \subseteq F(X)$ and the pair $\{T, G\}$ satisfies $\left(\operatorname{CLR}_{G}\right)$ property,

or

$S(X) \subseteq G(X)$ and the pair $\{S, F\}$ satisfies $\left(\mathrm{CLR}_{F}\right)$ property.

Then $S, T, F$, and $G$ have a unique common fixed point.

Proof. Without loss of generality, we assume that $T(X) \subseteq$ $F(X)$ and the pair $\{T, G\}$ satisfies $\left(\operatorname{CLR}_{G}\right)$ property; then there exists some sequence $\left\{x_{n}\right\}$ in $X$ such that $T x_{n}$ and $G x_{n}$ converge to $G x$, for some $x$ in $X$ as $n \rightarrow \infty$.

Since $T(X) \subseteq F(X)$ there exists a sequence $\left\{y_{n}\right\}$ in $X$ such that $T x_{n}=F y_{n}$; hence $F y_{n} \rightarrow G x$ as $n \rightarrow \infty$.

Now, we claim that $\lim _{n \rightarrow \infty} S y_{n}=G x$.

Let $\lim _{n \rightarrow \infty} S y_{n}=z$.

Taking $x=y_{n}$ and $y=x_{n}$ in (3), we have

$$
\begin{aligned}
& d\left(S y_{n}, T x_{n}\right) \\
& \quad \leq \phi\left(\max \left\{d\left(F y_{n}, G x_{n}\right), d\left(F y_{n}, S y_{n}\right), d\left(G x_{n}, T x_{n}\right)\right\}\right) .
\end{aligned}
$$

Letting $n \rightarrow \infty$, we have

$$
\begin{gathered}
d(z, G x) \leq \phi(\max \{d(G x, G x), d(G x, z), d(G x, G x)\}) \\
=\phi(d(G x, z))<d(z, G x), \\
\text { implies that } z=G x .
\end{gathered}
$$

Subsequently, we have $T x_{n}, G x_{n}, F y_{n}$, and $S y_{n}$ that converge to $z$.

Now, we claim that $T x=z$.

Taking $x=y_{n}$ and $y=x$ in (3), we have

$$
\begin{aligned}
& d\left(S y_{n}, T x\right) \\
& \quad \leq \phi\left(\max \left\{d\left(F y_{n}, G x\right), d\left(F y_{n}, S y_{n}\right), d(G x, T x)\right\}\right) .
\end{aligned}
$$

Letting $n \rightarrow \infty$, we have

$$
\begin{aligned}
d(z, T x) & \leq \phi(\max \{d(G x, G x), d(z, z), d(z, T x)\}) \\
& =\phi(d(z, T x))<d(z, T x), \\
& \text { implies that } z=T x=G x .
\end{aligned}
$$

Since the pair $(T, G)$ is weakly compatible, it follows that $T z=$ $G z$.

Also, since $T(X) \subseteq F(X)$, there exists some $y$ in $X$ such that $T x=F y(=z)$.

We next show that $F y=S y(=z)$.

Taking $y=x_{n}, x=y$ in (3), we have

$$
\begin{aligned}
& d\left(S y, T x_{n}\right) \\
& \quad \leq \phi\left(\max \left\{d\left(F y, G x_{n}\right), d(F y, S y), d\left(G x_{n}, T x_{n}\right)\right\}\right) .
\end{aligned}
$$

Letting $n \rightarrow \infty$, we have

$$
\begin{aligned}
d(S y, z) \leq & \phi(\max \{d(z, z), d(z, S y), d(z, z)\}) \\
& =\phi(d(z, S y))<d(S y, z), \\
& \text { implies that } S y=z=F y .
\end{aligned}
$$

But the pair $\{S, F\}$ is weakly compatible; it follows that $S z=$ $\mathrm{Fz}$.

Next, we claim that $S z=T z$.

Taking $x=z, y=z$ in (3), we have

$$
\begin{aligned}
d(S z, T z) \leq & \phi(\max \{d(F z, G z), d(F z, S z), d(G z, T z)\}) \\
& =\phi(d(S z, T z)) \\
& <d(S z, T z), \quad \text { implies that } S z=T z, \\
& \quad \text { that is, } S z=T z=F z=G z .
\end{aligned}
$$

Now, we show that $z=S z$.

Taking $x=z, y=x$ in (3), we have

$$
\begin{aligned}
d(S z, T x) & \leq \phi(\max \{d(F z, G x), d(F z, S z), d(G x, T x)\}) \\
& =\phi(d(S z, z))<d(S z, z),
\end{aligned}
$$

implies that $z=S z=T z=F z=G z$.

Hence $z$ is the common fixed point of $S, T, F$, and $G$.

If $S z=T z=F z=G z=z$ and $S t=T t=F t=G t=t$, then (3) gives

$$
\begin{aligned}
& d(S z, T t) \leq \phi(\max \{d(F z, G t), d(F z, S z), d(G t, T t)\}) \\
&=\phi(d(S z, T t)) \\
&<d(S z, T t), \quad \text { implies that } S z=T t, \\
& \text { that is, } z=t .
\end{aligned}
$$

Therefore, uniqueness follows.

Theorem 15. Let $(X, d)$ be Hausdorff and complete g.m.s. and let $\phi: \mathbb{R}^{+3} \rightarrow \mathbb{R}^{+}$be a $\mathcal{S}$ function. Let $S, T, F$, and $G$ be selfmaps on $X$ satisfying (5), (6), and (38). Then $S, T, F$, and $G$ have a unique common fixed point. 
Proof. Without loss of generality, we assume that $T(X) \subseteq$ $F(X)$ and the pair $\{T, G\}$ satisfies $\left(\mathrm{CLR}_{G}\right)$ property; then there exists some sequence $\left\{x_{n}\right\}$ in $X$ such that $T x_{n}$ and $G x_{n}$ converge to $G x$, for some $x$ in $X$ as $n \rightarrow \infty$.

Since $T(X) \subseteq F(X)$ there exists a sequence $\left\{y_{n}\right\}$ in $X$ such that $T x_{n}=F y_{n}$; hence $F y_{n} \rightarrow G x$ as $n \rightarrow \infty$.

Now, we claim that $\lim _{n \rightarrow \infty} S y_{n}=G x$.

Let $\lim _{n \rightarrow \infty} S y_{n}=z$.

Taking $x=y_{n}$ and $y=x_{n}$ in (5), we have

$d\left(S y_{n}, T x_{n}\right) \leq \phi\left(d\left(F y_{n}, G x_{n}\right), d\left(F y_{n}, S y_{n}\right), d\left(G x_{n}, T x_{n}\right)\right)$

Letting $n \rightarrow \infty$, we have

$$
\begin{aligned}
d(z, G x) & \leq \phi(d(G x, G x), d(G x, z), d(G x, G x)) \\
& =\phi(0, d(G x, z), 0) \\
& <d(z, G x), \quad \text { implies that } z=G x .
\end{aligned}
$$

Subsequently, we have $T x_{n}, G x_{n}, F y_{n}$, and $S y_{n}$ that converge to $z$.

Now, we claim that $T x=z$.

Taking $x=y_{n}$ and $y=x$ in (5), we have

$$
d\left(S y_{n}, T x\right) \leq \phi\left(d\left(F y_{n}, G x\right), d\left(F y_{n}, S y_{n}\right), d(G x, T x)\right)
$$

Letting $n \rightarrow \infty$, we have

$$
\begin{aligned}
d(z, T x) & \leq \phi(d(G x, G x), d(z, z), d(z, T x)) \\
& =\phi(0,0, d(z, T x)) \\
& <d(z, T x), \quad \text { implies that } z=T x=G x .
\end{aligned}
$$

Since the pair $(T, G)$ is weakly compatible, it follows that $T z=$ $G z$.

Also, since $T(X) \subseteq F(X)$, there exists some $y$ in $X$ such that $T x=F y(=z)$.

We next show that $F y=S y(=z)$.

Taking $y=x_{n}, x=y$ in (5), we have

$$
d\left(S y, T x_{n}\right) \leq \phi\left(d\left(F y, G x_{n}\right), d(F y, S y), d\left(G x_{n}, T x_{n}\right)\right) .
$$

Letting $n \rightarrow \infty$, we have

$$
\begin{array}{r}
d(S y, z) \leq \phi(d(z, z), d(z, S y), d(z, z)) \\
=\phi(0, d(z, S y), 0)<d(S y, z), \\
\text { implies that } S y=z=F y .
\end{array}
$$

But the pair $\{S, F\}$ is weakly compatible; it follows that $S z=$ $\mathrm{Fz}$.

Next, we claim that $S z=T z$.

Taking $x=z, y=z$ in (5), we have

$$
\begin{aligned}
& d(S z, T z) \leq \phi(d(F z, G z), d(F z, S z), d(G z, T z)) \\
&=\phi(d(S z, T z), 0,0)<d(S z, T z), \\
& \text { implies that } S z=T z .
\end{aligned}
$$

Now, we show that $z=S z$.

Taking $x=z, y=x$ in (5), we have

$$
\begin{aligned}
d(S z, T x) & \leq \phi(d(F z, G x), d(F z, S z), d(G x, T x)) \\
& =\phi(d(S z, z), 0,0)<d(S z, z),
\end{aligned}
$$

implies that $z=S z=T z=F z=G z$.

Hence $z$ is the common fixed point of $S, T, F$ and $G$.

If $S z=T z=F z=G z=z$ and $S t=T t=F t=G t=t$, then (5) gives

$$
\begin{aligned}
d(S z, T t) \leq & \phi(d(F z, G t), d(F z, S z), d(G t, T t)) \\
= & \phi(d(S z, T t), 0,0)<d(S z, T t) \\
& \text { implies that } S z=T t \text { or } z=t .
\end{aligned}
$$

Therefore, $z=t$ and the common fixed point is unique.

\section{Acknowledgment}

One of the authors (S. Kumar) is very grateful to UGC for providing MRP under Ref. F. no. 39-41/2010(SR).

\section{References}

[1] A. Branciari, "A fixed point theorem of Banach-Caccioppoli type on a class of generalized metric spaces," Publicationes Mathematicae Debrecen, vol. 57, no. 1-2, pp. 31-37, 2000.

[2] G. Jungck, "Compatible mappings and common fixed points," International Journal of Mathematics and Mathematical Sciences, vol. 9, no. 4, pp. 771-779, 1986.

[3] G. Jungck and B. E. Rhoades, "Fixed points for set valued functions without continuity," Indian Journal of Pure and Applied Mathematics, vol. 29, no. 3, pp. 227-238, 1998.

[4] M. Aamri and D. El Moutawakil, "Some new common fixed point theorems under strict contractive conditions," Journal of Mathematical Analysis and Applications, vol. 270, no. 1, pp. 181$188,2002$.

[5] W. Sintunavarat and P. Kumam, "Common fixed point theorems for a pair of weakly compatible mappings in fuzzy metric spaces," Journal of Applied Mathematics, vol. 2011, Article ID 637958, 14 pages, 2011.

[6] C. M. Chen, "Common fixed-point theorems in complete generalized metric spaces," Journal of Applied Mathematics, vol. 2012, Article ID 945915, 14 pages, 2012.

Hence, $S z=T z=F z=G z$. 


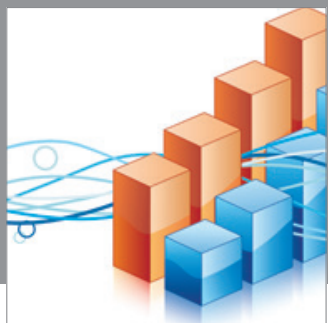

Advances in

Operations Research

mansans

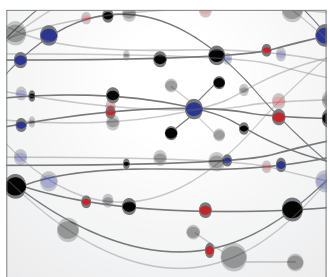

The Scientific World Journal
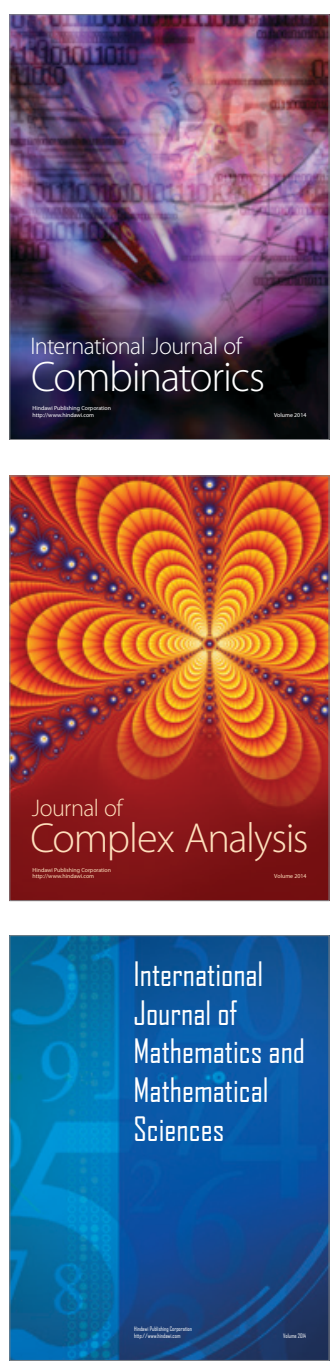
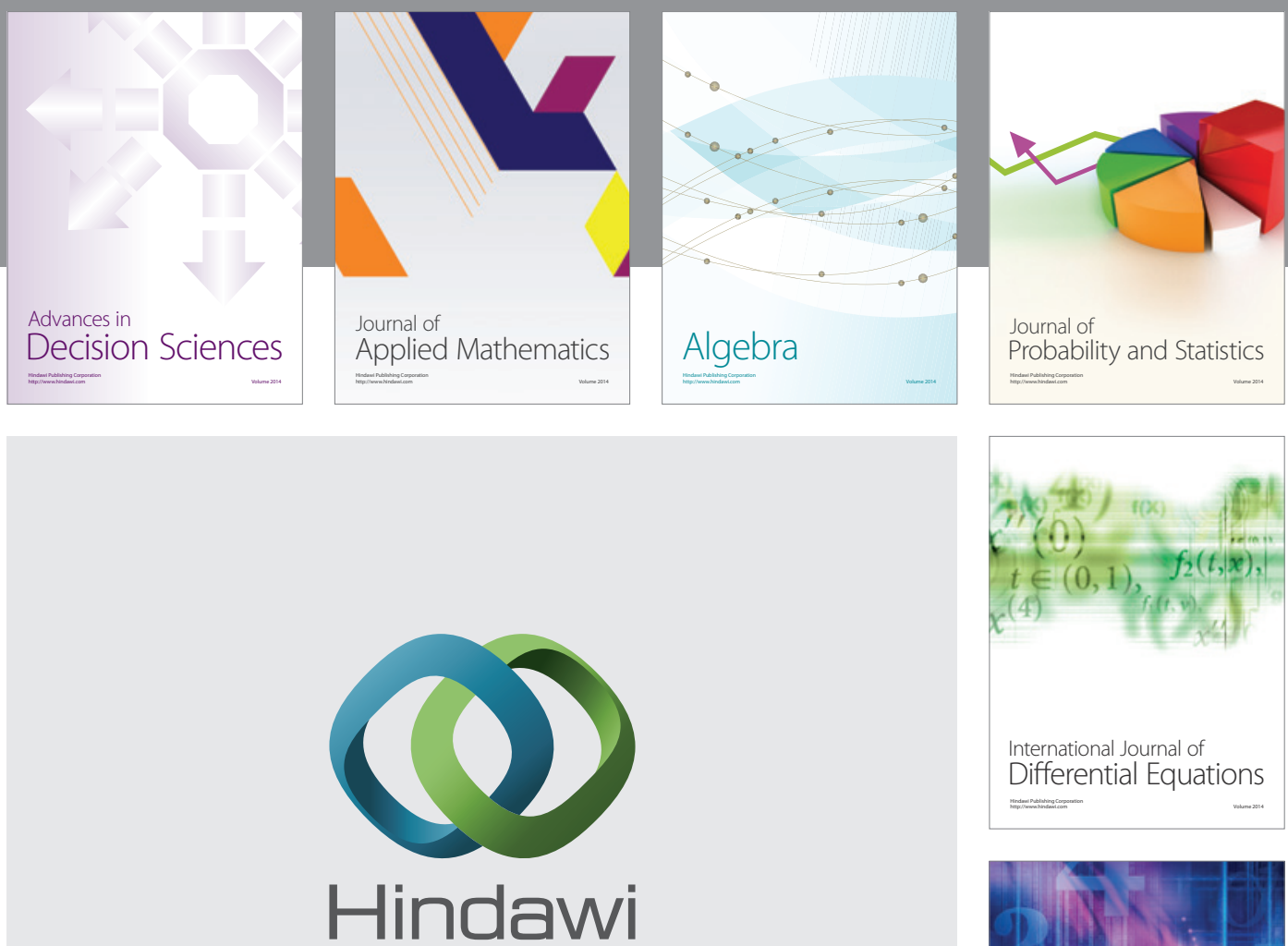

Submit your manuscripts at http://www.hindawi.com
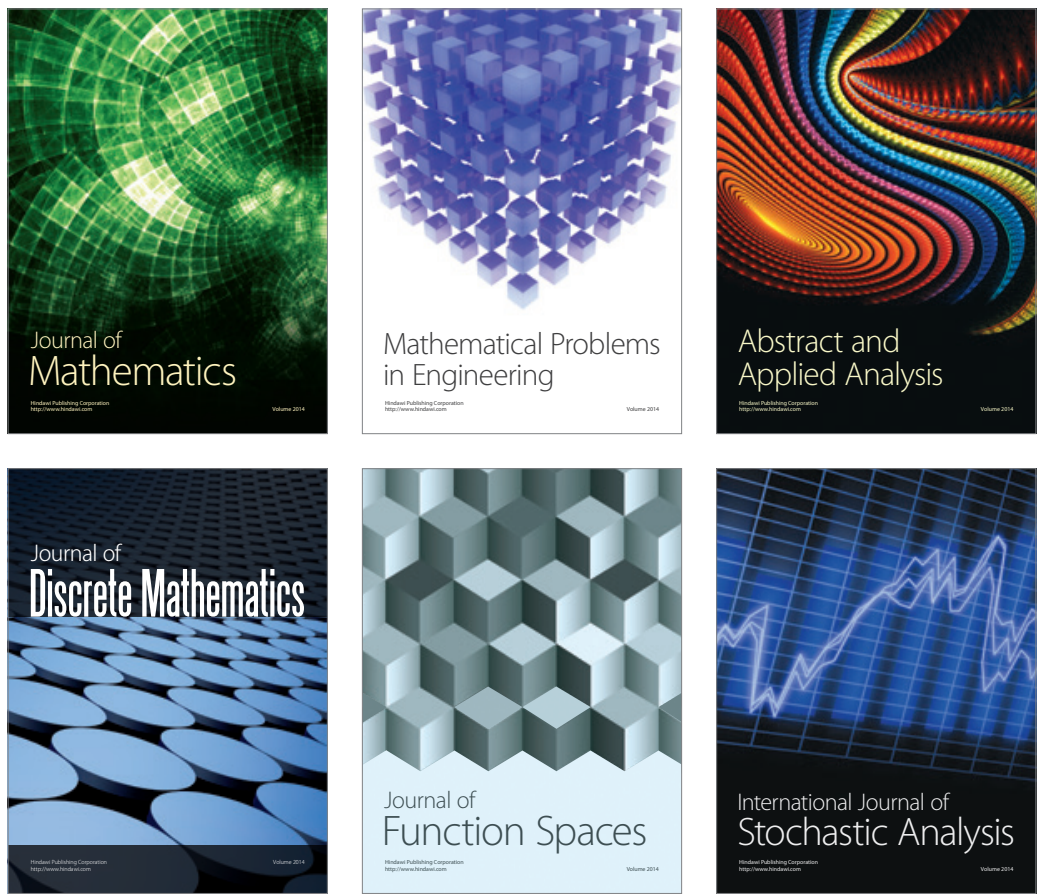

Journal of

Function Spaces

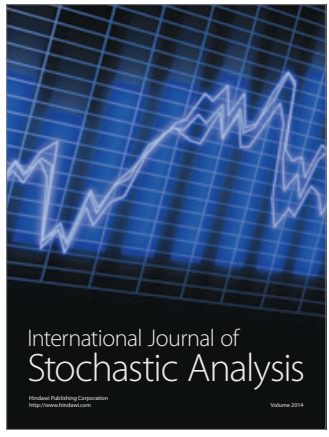

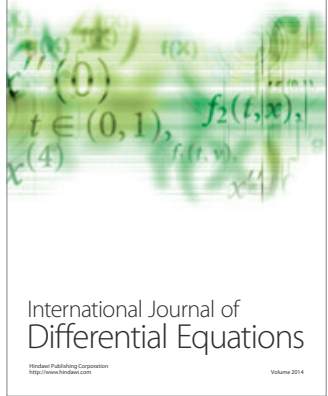
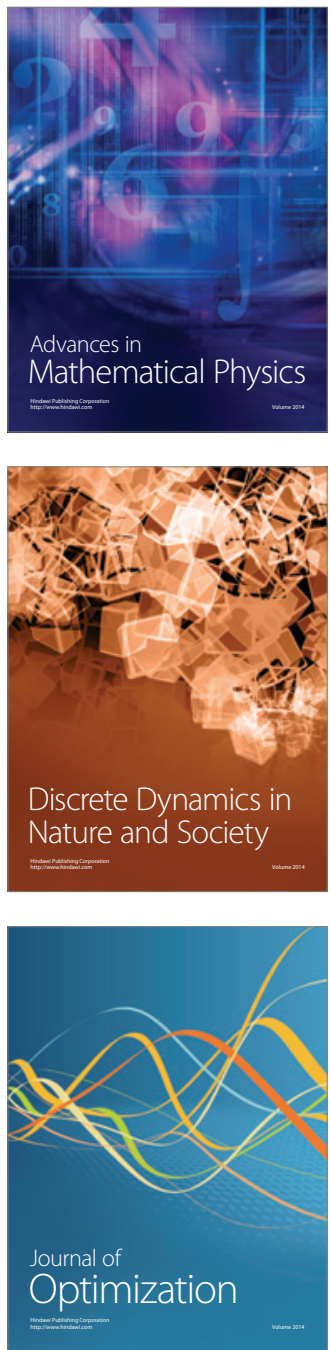